\title{
Le genre des controverses : approches féministes et queer
}

Maxime Cervulle et Virginie Julliard

\section{Q OpenEdition}

1 Journals

Édition électronique

URL : https://journals.openedition.org/questionsdecommunication/12076

DOI : 10.4000/questionsdecommunication. 12076

ISSN : 2259-8901

Éditeur

Presses universitaires de Lorraine

\section{Édition imprimée}

Date de publication : 1 septembre 2018

Pagination : 7-22

ISBN : 978-2-8143-0519-9

ISSN : $1633-5961$

\section{Référence électronique}

Maxime Cervulle et Virginie Julliard, « Le genre des controverses : approches féministes et queer », Questions de communication [En ligne], 33 | 2018, mis en ligne le 01 septembre 2018, consulté le 21 septembre 2021. URL : http://journals.openedition.org/questionsdecommunication/12076 ; DOI : https://doi.org/10.4000/questionsdecommunication.12076 
MAXIME CERVULLE

Centre d'études sur les médias, les technologies et l'internationalisation

Université Paris 8 Vincennes - Saint-Denis

F-93526

maxime.cervulle@univ-paris8.fr

VIRGINIE JULLIARD

Connaissance, organisation et systèmes techniques

Université de technologie de Compiègne

F-60203

virginie.julliard@utc.fr

\section{LE GENRE DES CONTROVERSES : APPROCHES FÉMINISTES ET QUEER}

$\longrightarrow$

urant les vingt dernières années, plusieurs controverses ont ouvert un espace de conflictualité autour de la définition et des usages politiques de la « différence des sexes ». Les controverses relatives à la loi sur la « parité », au Pacs, au port du « voile » et de la « burqa », au « mariage pour tous » ou encore aux plans d'action publique pour l'égalité filles-garçons à l'école ont toutes participé d'une mise en délibération des conceptions hégémoniques des identités de genre et des rapports sociaux qui les sous-tendent. L'instrumentalisation de l'expression « théorie du genre » à partir de 201 I témoigne ainsi d'une radicalisation des positions, avec la montée d'une « panique morale » exprimant l'inquiétude de voir disparaître l'altérité sexuelle et les rôles sociaux qui lui sont liés. Les controverses constituent un objet privilégié pour saisir le caractère normatif d'un espace public où les rôles sociaux se redistribuent sous contrainte, dans la mesure où elles ouvrent des séquences de délibération publique mobilisant une multitude d'acteurs et d'actrices différemment positionné e.s dans la formation sociale. Elles donnent à voir, dans un même mouvement, les dynamiques de transformation et de reproduction non seulement de l'espace public, mais des rapports de genre. 
L'entreprise d'historicisation du sexe comme objet scientifique, engagée dans le champ de l'épistémologie et de la philosophie féministe des sciences, a démontré l'instabilité définitionnelle du sexe et la difficulté, si ce n'est l'impossibilité, à fonder scientifiquement la bicatégorisation par sexe (Kraus, 2000). Surtout, elle a souligné à quel point la crise constitue le régime théorique ordinaire du sexe (Dorlin, 2005). Au sein de l'histoire de la recherche biologique et médicale, la théorie de la bicatégorisation sexuée est en effet perpétuellement en crise : sujette à des remises en cause et révisions qui se manifestent par l'émergence constante de nouveaux critères de détermination du sexe des individus (par exemple le sexe dit « hormonal », « chromosomique », 《 gonadique », « phénotypique » ou encore « génique »). La fragilité épistémique de chacun de ces critères, autant que la valse des révisions théoriques qu'ils ont impliquée face aux cas empiriques qui démentent leur validité, donnent à penser que « la crise [comme régime théorique] est l'expression même de l'historicité d'un rapport de domination qui se modifie, mute et doit constamment redéfinir son système catégoriel pour assurer les conditions de sa reproduction » (ibid. : I 34). En transposant cette idée selon laquelle la crise peut opérer comme un moyen d'assurer la pérennité d'un système de représentations sociales et de l'ordre qu'il participe à fonder, nous proposons de considérer les multiples controverses relatives au genre et à la sexualité qui se sont déployées dans l'espace public contemporain moins comme des signes d'affaiblissement de l'ordre de genre que comme des temps de reconfiguration permettant de garantir son maintien. Selon cette perspective, penser le genre à partir des controverses permet de donner à voir le rôle joué par la délibération politique et la médiation médiatique dans la reproduction des rapports et des identités de genre. Cette reproduction opère cependant moins comme une reconduction à l'identique que selon un principe d'itérabilité (Derrida, 1989 ; Butler, 1993), où chaque répétition de la marque du genre la modifie et l'altère. La plasticité politique du genre dans l'espace médiatique, tout comme la plasticité épistémique du sexe dans le champ biomédical, sont une condition de leur permanence aussi bien que de leur possible contestation publique.

Treize ans après la parution de la septième livraison de Questions de communication qui accueillait le dossier « Espaces politiques au féminin » (Fleury, Walter, 2005), ce dossier sur « le genre des controverses » entend contribuer au champ de réflexion ouvert au sein des sciences de l'information et de la communication (SIC) autour de la question de l'articulation entre genre, médias et espace public. Les recherches sur le genre dans les SIC, qui se sont particulièrement développées à la suite des débats relatifs au Pacs et à la parité, sont désormais bien implantées'.

\footnotetext{
' Le nombre et la régularité des dossiers consacrés au genre publiés dans les revues de la discipline depuis 2003 en témoignent: « Une communication sexuée ? 》 (Bonnafous, Jouët, Rieffel, 2003) ; « Sexe \& Communication » (Bouchard, Froissart, 2004) ; « Espaces politiques au féminin » (Fleury, Walter, 2005); « Usages politiques du genre » (Desmarchelier, Rennes, 2005) ; « Femmes et médias : le 8 mars à la Une » (Coulomb-Gully, Bonnafous, 2007); « Penser le genre en sciences de l'information et de la communication et au-delà » et « Penser le genre en sciences de l'information et de la communication et au-delà (2) » (Fleury, Walter, 2009a ; 2009b) ; « Médias : la fabrique du Genre » (Coulomb-Gully, 20I I) ; « Les langages du genre : sémiotique et communication » (Berthelot-Guiet, Kunert, 20 I3); « Le
} 
Elles interrogent la production du genre dans le discours de presse (Tavernier, 2004 ; Julliard, 2012 ; Olivesi, 2012 ; Dalibert, 2013 ; Cervulle, Julliard, 20I3) ou sur les réseaux socio-numériques (par exemple dans les débats qui se déploient sur Twitter [Cervulle, Pailler, 2014 ; Julliard, 20 I6]). Elles étudient l'effet des rapports de genre sur les contraintes organisationnelles du journalisme (Damian-Gaillard, Saïtta, 201 I ; Chauvel, Le Renard, 20 I3), la définition institutionnelle du genre (Hernández Orellana, Kunert, 2014) ou encore les représentations médiatiques comme lieu de recomposition des identités de genre (Coulomb-Gully, Méadel, 2012 ; Quemener, 2014 ; Damian-Gaillard, Montañola, Olivesi, 2014 ; Lécossais, 2014 ; Espineira, 20 I5). Elles se penchent également sur les modes de régulation sociale de la sexualité tels qu'ils peuvent se saisir dans les médias. C'est le cas par exemple des recherches de Fred Pailler (20 I I) sur les modes de sexualisation des dispositifs numériques ou de Florian Vörös (20I5) sur les usages genrés de la pornographie en ligne.

Les nombreux travaux en SIC qui problématisent le genre manifestent la labilité du concept. Deux acceptions se côtoient en effet dans ces travaux - un fait qui n'est pas propre aux SIC, mais que l'on retrouve dans l'ensemble des sciences humaines et sociales (Marignier, 2016 :37-40). Le genre y désigne I) un rapport social établissant des différences entre hommes et femmes et les rendant économiquement fonctionnelles, ainsi que 2) les identités que ce rapport fait émerger, soit les multiples formes d'expression du masculin et du féminin qui actualisent leur version normative idéalisée. Surtout, ces travaux s'appuient sur différentes conceptualisations de la relation entre sexe et genre, qui manifestent différents degrés de constructivisme. Le genre peut ainsi être conçu comme une mise en forme culturelle de ce substrat biologique que serait le sexe ou bien comme la manière par laquelle la catégorie de sexe elle-même est socialement construite par la reproduction discursive continue du genre (voir notamment Butler, 1990 ; De Lauretis, 1987)². Cette seconde position ouvre des perspectives particulièrement intéressantes pour les SIC. En conférant une place déterminante à la performativité du genre, aux effets de signification et à la politique des représentations, cette position situe l'étude des médias, du discours et des débats publics au premier plan pour la compréhension des transformations historiques de la « différence des sexes », des partages sociaux qu'elle recouvre et de leurs usages politico-économiques.

Le présent dossier accompagne ce mouvement de réflexion autour des rapports entre genre, médias et politique en mettant la question des controverses à l'épreuve des outils critiques forgés à l'intersection des SIC, des études de genre mais aussi des études queer. Si les études de genre infusent nombre de travaux actuels en SIC, les apports des études queer restent encore peu mobilisés. Nées aux ÉtatsUnis sous la double impulsion d'un féminisme d'inspiration poststructuraliste et

genre dans la communication et les médias » (Julliard, Quemener, 20 I4) ; « Sexualités » (Amato, Pailler, Schafer, 2014) ; « La pornographie et ses discours » (Paveau, Perea, 2014) ou encore « Écrire le genre » (Constantin de Chanay, Chevalier, Gardelle, 2017).

2 Pour une version matérialiste de l'idée selon laquelle, loin de traduire le sexe, le genre le produirait, voir les travaux de Christine Delphy (200I). 
de travaux en histoire portant sur l'émergence et la circulation des identités sexuelles, les études queer poursuivent l'entreprise de dénaturalisation du sexe, du genre et de la sexualité engagée par les théories féministes. Un projet qui implique d'interroger les formes et fonctions de la régulation de la sexualité dans l'espace public, parallèlement à la critique féministe de l'androcentrisme de l'espace public et des théories qui le conceptualisent.

\section{Faire et défaire les publics des controverses}

Un point central de l'analyse des controverses consiste à identifier les publics autour desquels et pour lesquels elles s'organisent. Comme le souligne Cyril Lemieux (2007 : 195), les controverses « ont toujours une structure triadique: [elles] renvoient à des situations où un différend entre deux parties est mis en scène devant un public, tiers placé dès lors en position de juge ». La définition idéal-typique de la controverse que propose Cyril Lemieux - qui, à ce titre, ne recouvre qu'imparfaitement les cas empiriques - insiste sur l'égale compétence de ces trois instances comme caractéristique discriminante.

«Plus le public placé en position de juger d'un différend est strictement composé de pairs, c'est-à-dire d'individus auxquels chaque partie en désaccord peut reconnaître la même compétence distinctive au jugement que celle qu'elle se reconnait, plus nous semblons disposés à parler de "controverse" » (ibid.: 196).

Nombre de controverses sont soumises à un processus d'élargissement, d'extension hors de leur champ initial de référence ; c'est toutefois l'essor du différend au sein d'un milieu spécialisé et relativement autonome qui permettrait selon Cyril Lemieux de parler de controverse. Saisies dans leur diversité, les controverses seraient cependant soumises à la publicisation selon différents degrés (allant du confinement de la controverse à son champ de référence jusqu'à sa médiatisation la plus intensive). De la même façon, le degré de spécialisation du public constitué par une controverse varierait fortement, les deux figures limites étant le public de pairs et le public profane. Cyril Lemieux souligne enfin la nécessité, pour qui souhaiterait rendre compte de la dimension instituante d'une controverse - c'est-à-dire de sa capacité à transformer les institutions et les acteurs qui y sont impliqués -, de prendre pour point de départ « l'espace institutionnel ou [le] milieu social où elle est née plutôt que [...] la scène médiatique où elle a été rendue visible au plus grand nombre » (ibid. : 201).

Les controverses traitées dans ce dossier ont toutes un fort degré de visibilité médiatique - lorsqu'elles ne sont pas directement issues d'un processus conflictuel interne à l'espace médiatique, comme c'est par exemple le cas du conflit entre journalistes lié au traitement de l'ouvrage Pour en finir avec Eddy Bellegueule et au statut à lui accorder, étudié par Marion Dalibert. Les contributions à ce dossier prennent par ailleurs le contrepied de la préconisation méthodologique de Cyril Lemieux, en partant de la médiation médiatique plutôt que des espaces institutionnels où les controverses trouveraient leur origine. En effet, il s'agit moins, pour ces auteur.e.s, 
de donner à voir l'essor et la circulation de ces controverses que de saisir la transformation idéologique qu'elles impliquent, les tensions discursives nouvelles qu'elles mettent en jeu et qui participent d'une réélaboration des antagonismes politiques à partir de dispositifs de médiation : le débat télévisé et ses réceptions dans les plateformes numériques et la presse en ligne pour Florian Vörös ; la presse, la radio et la télévision pour Marion Dalibert ; les festivals dans la contribution de Konstantinos Eleftheriadis. Dans une perspective un peu différente, la proposition de Fatima Khemilat étudie la manière par laquelle le droit est devenu l'interface privilégiée de la gestion de la visibilité féminine du fait religieux musulman dans l'espace public, tandis que celle de Nelly Quemener expose les enjeux méthodologiques que revêt l'étude des « intensités affectives » propres à la médiation médiatique des controverses. Si, dans ces textes, l'enjeu est bien d'apprécier la fonction instituante et donc performative des controverses, et non d'y voir l'expression mécanique d'une structure sociale sous-jacente, c'est moins pour mettre l'accent sur une réorganisation institutionnelle proprement dite que sur l'émergence par leur biais de nouveaux modes d'interpellation des publics, de nouveaux rapports au monde et de nouveaux régimes de sensibilité. Ce dernier point est ainsi au cœur de la contribution de Nelly Quemener : en insistant sur la dimension affective de la controverse, elle donne à voir combien celle-ci opère comme un « processus social auto-réalisateur ». La redistribution de valeurs et émotions autour de l'objet de la controverse constitue sa «force de dichotomisation » et nourrit les injonctions à « se positionner » dans ce cadre binaire. Un tel processus ferait émerger, selon elle, des modes de subjectivation spécifiques : la possibilité, autant pour les acteurs et actrices de la controverse que pour ses publics, de donner des gages de « respectabilité » et d'affiliation à un groupe donné en répondant à l'interpellation médiatique formulée en termes de valeurs et d'émotions ${ }^{3}$.

On peut ainsi penser que les controverses ont la capacité à faire et défaire des publics - ces derniers pouvant d'ailleurs être saisis comme l'entité concrète qui se manifeste en réception aussi bien que comme une figure convoquée plus ou moins explicitement dans le discours (Warner, 2005 : 67). Le type de public qu'une controverse fait émerger comme instance tierce, les registres discursifs au travers desquels il est interpellé et la fonction qui lui est assignée sont ainsi autant de manières d'apprécier le processus conflictuel et sa portée. Les controverses fortement médiatisées ont d'ailleurs ceci de singulier qu'elles peuvent construire leur adresse à différents publics, en fonction des supports et de leurs propres publics imaginés, ce qui implique des cadrages différents du conflit. Surtout, elles reposent moins sur l'égale compétence des parties en confrontation que sur un principe de disqualification de l'une des positions, généralement celle jugée la moins fédératrice ou la plus éloignée du public imaginé du média qui se trouve alors renvoyé à un état d'incompétence ou d'illégitimité (Cervulle, 2017). Aussi peut-on considérer que la proposition de Cyril Lemieux selon laquelle une controverse se caractériserait

\footnotetext{
3 Sur la question des valeurs, voir les échanges des précédentes livraisons de Questions de communication (Heinich, 2017 ; Fleury, Walter, 2017 ; Kaufmann, Gonzalez, 2017 ; Quéré, 2017 ; Martuccelli, 2017).
} 
par une structure triadique où les trois acteurs seraient symboliquement dotés d'une égale compétence relève d'une forme de délibération idéale, rarement rencontrée empiriquement, en particulier lorsque les controverses engagent la mise en cause ou la conservation des rapports sociaux liés au genre, à la race, à la classe ou au partage et à la hiérarchisation des sexualités. Dans l'espace médiatique, la controverse semble précisément prendre appui sur un processus en apparence paradoxal de disqualification de l'une des parties, en fonction du public imaginé du support médiatique ${ }^{4}$. Tout en mettant en scène la confrontation entre les parties, la structuration médiatique de la controverse consiste à ne pas les faire apparaître tout à fait comme des pairs. On peut en dire autant du public imaginé occupant la fonction de « juge » de la controverse. Les controverses sont des moments de réorganisation discursive des publics, où l'enjeu réside sans doute autant dans la résolution du conflit par la délibération que dans la distribution inégale de la compétence, de l'expertise et de la capacité (à dire ou à faire) de différents publics. Comme le souligne le théoricien queer Michael Warner (2005), le type de public qu'une controverse fait émerger comme instance tierce, les registres discursifs au travers desquels il est interpellé et la fonction qui lui est assignée sont donc autant de manières d'apprécier le processus conflictuel.

\section{Critiques féministes et queer de l'espace public}

La critique féministe de la théorie habermassienne de l'espace public montre, de son côté, combien le genre structure celui-ci notamment à travers des conditions de participation politique inégales et l'établissement d'une ligne de partage entre « public » et « privé ». Nancy Fraser ( $1985: 56)$ a ainsi démontré que « la structure institutionnelle de [l'espace public] est réalisée au moyen de rôles sociaux genrés ». La domination masculine se manifeste dans des inégalités matérielles qui biaisent les conditions d'égale participation, elle s'exprime dans l'impossibilité d'un dialogue entre pairs et la disqualification de la parole des femmes, ainsi que dans le codage masculin de la citoyenneté 5 . II s'ensuit que les conditions d'une communication démocratique ne sont pas remplies. Pour combattre les injustices sexistes, Nancy Fraser considère qu'il faut considérer les inégalités de répartition (les théories de la justice distributive sont appelées à considérer la pauvreté qui résulte de

\footnotetext{
${ }^{4}$ La disqualification de l'une des parties peut avoir des conséquences sur la reterritorialisation de formations discursives propres à certaines des parties en présence vers d'autres scènes publiques où elles sont plus audibles: par exemple le Web et les plateformes numériques plutôt que les « médias traditionnels » pour les opposants à ladite « théorie du genre » (Julliard, 20 I7).

${ }^{5}$ Le codage masculin de la citoyenneté fait dire à Carole Pateman (1988) que le contrat social est en réalité un « contrat sexuel ». Avant C. Pateman et N. Fraser, Jean Elshtain (198I) a remis en question le caractère soi-disant neutre de la citoyenneté, plaidant pour que les valeurs maternelles du soin viennent remplacer la vision participative et républicaine de la citoyenneté. Dans le sillage de N. Fraser, les réflexions sur la dimension genrée de l'espace public se sont multipliées (Lunt, Livingstone, 1994 ; Göle, 1997 ; Rendall, 1999), ouvrant un large champ de problématisation des rapports entre inégalités sociales, identités de genre et citoyenneté.
} 
la division sexuée du travail, par exemple) et les inégalités de reconnaissance (la philosophie de la reconnaissance (voir Taylor, 1994 ; Honneth, 1992) est invitée à se pencher, notamment, sur la construction juridique de la vie privée ${ }^{6}$ ou sur l'impérialisme culturel produit par l'institutionnalisation des modèles d'appréciation androcentrés dans le droit ou la médecine). La répartition des ressources matérielles entre toutes et tous, qui garantit l'indépendance d'esprit, d'une part, et l'institutionnalisation de modèles d'appréciation alternatifs qui assure un respect identique aux individus, d'autre part, autorise à chacune et chacun d'interagir en tant que pairs. C'est ce que Nancy Fraser (2010) appelle « parité participative ${ }^{7}$ ». Qui plus est, la philosophe d'inspiration francfortienne (Fraser, 1992 : 144- |45) établit que les termes « public » et « privé » sont « des classifications culturelles et des étiquettes rhétoriques [qui,] employées dans un discours politique, [...] servent fréquemment à délégitimer certains intérêts, points de vue et sujets et à en mettre d'autres en valeur » (avec pour conséquence de perpétuer, au-delà des restrictions officielles sur la participation politique, « les handicaps socio-sexués et de classe »). À cet égard, elle dénonce les usages idéologiques d'une rhétorique de la vie privée qui soustrait les problèmes « économiques 》 et «familiaux 》 du champ de la contestation sociale. Audrey Benoît (2014 : 126) défend l'intérêt d'un approfondissement constructiviste de cette position :

«"La rhétorique de la vie privée domestique" est la forme par laquelle, mais aussi dans laquelle des contenus thématiques objectifs [...] sont exclus du débat public. Or un tel constat appelle une analyse constructiviste : comment ces modalités discursives formelles produisent-elles leurs propres objets ? Comment cette "rhétorique" crée-t-elle les conditions même de la distinction du "public" et du "privé" ?8 $\gg$.

Aussi Audrey Benoît considère-t-elle que la déconstruction de la « publicité » passe par l'identification de la manière par laquelle le discours matérialise des effets de pouvoir. La contribution à ce dossier de Fatima Khemilat, qui porte sur les controverses françaises autour du voile et sur le fondement des changements législatifs auxquels elles ont donné lieu, s'inscrit dans cette perspective. À propos de la controverse sur le « voile intégral », elle montre par exemple combien la définition de l'ordre public au principe de l'interdiction ne prend sens que dans le partage public/privé qu'instaure le « régime de visibilité républicain », ce dernier reléguant les signes religieux ostensibles dans la sphère privée et exigeant la disponibilité du visage aux regards au nom de la « moralité publique » et de la « dignité humaine ».

\footnotetext{
${ }^{6}$ Au sujet de la construction juridique de la vie privée, voir également les travaux d'Anita Allen (1988) sur la structuration genrée de la vie privée et les restrictions qui ont historiquement pesé sur l'autonomie personnelle (et notamment corporelle) des femmes.

${ }^{7}$ La parité participative se distingue de la « parité française », telle que la comprend l'auteure : I) parce qu'elle est qualitative, plutôt que quantitative ; 2 ) parce qu'elle intègre les deux dimensions de la justice sociale et non la seule dimension de la reconnaissance ; 3) parce qu'elle déborde le seul champ du politique ; 4) parce qu'elle permet d'intégrer d'autres axes de différenciation sociale au-delà du seul sexe ; et 5) parce qu'elle garantit une possibilité de parité, plutôt qu'elle ne vise une parité réelle.

${ }^{8}$ C'est l'auteure qui souligne.
} 
La théorie queer formule trois critiques à l'égard des propositions de Nancy Fraser : la première concerne les modalités d'inclusion, la dexuième a trait aux effets de l'usage thétorique des concepts de public et privé, la troisième porte sur la non-prise en compte du caractère normatif des modalités d'intervention dans le débat public. Selon la perspective queer, l'inclusion - ou la parité participative - ne règle pas totalement les problèmes soulevés par la structuration genrée de l'espace public. D'abord, parce que les « nouveaux et nouvelles entrant·e.s » seraient supposé.e.s porter la voix du groupe qu'ils/elles sont censé.e·s représenter, ce qui risquerait de réifier les différentes identités (alors même que chaque individu est porteur d'identités multiples et mouvantes, parfois contradictoires) $)^{9}$. La mise en place de la parité en France, par exemple, a été l'occasion de (re)produire le genre en politique : il était attendu que les femmes s'investissent dans certaines thématiques et promeuvent certaines valeurs soi-disant « féminines » (Dulong, Matonti, 2005 ; Achin et al., 2007 ; Julliard, 20 I2). Ensuite, parce que la participation politique et l'accès à l'espace public restent conditionnés par le consentement aux identités de genre normatives et au partage des sexualités (Clarke, 2000 ; Dalibert, Quemener, 2016 ; Cervulle, 2014 ; Espineira, $2014)^{10}$. C'est à la théorie queer que l'on doit d'avoir approfondi la compréhension des effets de la privatisation du sexe et de la sexualisation de la personnalité - dont Lauren Berlant et Michael Warner (1998) rappellent qu'elles ont été mises au jour par Jürgen Habermas (1962), d'une part, et par Michel Foucault (1976), d'autre part. Lauren Berlant et Michael Warner ont ainsi montré que la conception de la sexualité comme propriété subjective fonde le binarisme homo/hétérosexualité dans la mesure où elle « empêche la mise en délibération des arrangements sociaux fondés sur [c]e binarisme » (Cervulle, 2014 : |47). Ces auteure-s proposent, pour leur part, d'appréhender l'hétérosexualité comme une culture publique donnant forme à un public hégémonique. Ce dossier comporte la traduction de leur célèbre article de 1998 « Sex in Public » qui développe cette proposition.

Judith Butler (1997 : 179) interpelle quant à elle directement Nancy Fraser, estimant que cette dernière « ne cherche pas à connaître les exclusions nécessaires à la définition et à la naturalisation de la sphère de la reproduction ». En effet, en assumant l'hypothèse selon laquelle la politique queer, que Nancy Fraser (1997 ; 2010) interprète comme une lutte pour la reconnaissance des minorités sexuelles,

${ }^{9}$ La critique de l'inclusion a également été émise par Iris Marion Young. Selon la politiste, l'inclusion formelle ne suffit pas, car les inégalités structurelles sont reconduites jusque dans les procédures de délibération. Participer à ces procédures contribuerait alors à leur « conférer une légitimité imméritée et à contribuer à la censure des outsiders » (Young, 200 I : |45). I. M. Young (1990) prône alors une citoyenneté différenciée et une représentation des groupes sociaux opprimés. Cette représentation doit s'accompagner du droit de proposer des politiques fondées sur l'intérêt propre de ces groupes et d'un droit de véto à opposer aux politiques générales qui leur porteraient atteinte.

${ }^{10}$ Dans un autre registre, Chantal Mouffe (1999) promeut une conception agonistique de la démocratie, laquelle se fonde sur la reconnaissance du caractère irréductible du conflit des identités. II s'agit, pour la théoricienne, de constituer un nous, dans un contexte de diversité et de conflit, qui se distingue d'un eux (les adversaires qui acceptent de faire partie de l'espace agonistique et dont il s'agit de combattre les idées). L'inclusion n'a pas de sens dès lors qu'une distinction nous/eux compatible avec le pluralisme est établie. 
se situerait exclusivement dans le champ culturel, elle dénie la part matérielle de la régulation sociale de la sexualité, l'excluant de fait du champ de l'économie politique. Selon Judith Butler, l'auteure de Qu'est-ce que la justice sociale? (2005) reconduit, par là même, la division et la hiérarchisation des oppressions, selon qu'elles relèveraient totalement (classe et race), partiellement (genre), ou non (sexualité) du champ de l'économie politique. Judith Butler (1997 : 178) considère pour sa part que,

\footnotetext{
« [si] les luttes pour transformer le champ social de la sexualité ne sont pas perçues comme étant au cœur même de l'économie politique, ce n'est pas parce qu'elles [ne seraient pas] directement liées à des questions de travail, mais plutôt parce qu'elles ne peuvent pas se comprendre si la sphère économique elle-même n'est pas étendue de manière à inclure à la fois la reproduction des biens et la reproduction sociale des personnes $\gg$.
}

Comme le soulignent Karl Marx (I 846) et Friedrich Engels (I 884), la première production est celle d'êtres humains. Les luttes pour transformer le champ social de la sexualité témoignent que la distinction entre sphère culturelle et sphère économique ne tient pas : l'échange sexuel ayant des effets culturels aussi bien que matériels. L'étude de la constitution de contre-publics féministes, gays, lesbiens ou transgenres permet alors d'analyser la renégociation des limites du public et de l'intérêt général, autant que les jeux de reprivatisation de la sexualité (Floyd, 2009). En France, la controverse relative à l'ouverture du mariage aux couples de personnes du même sexe témoigne à la fois de cette porosité des sphères (l'ouverture du mariage est défendue au motif qu'elle autorise une égalité de droit, notamment patrimonial), du refus par certain·e·s acteur.rice·s de débattre publiquement des arrangements sexuels particuliers (lequel refus se justifie par une naturalisation de la famille, de la procréation et de la filiation), et de la hiérarchisation des luttes qui travaille la doxa (il y aurait des problèmes « plus importants à régler » que l'égalité des droits entre couples hétéro/homosexuels en France) (Cervulle, Julliard, 2013).

Enfin, la théorie queer s'interroge sur les modalités de prise de parole dans le débat public. Si Nancy Fraser (1992) a mis en avant l'existence d'une pluralité de publics et les rapports conflictuels entre ces publics, Michael Warner (2005 : I I8I19) attire l'attention sur le fait que la théoricienne ne semble pas se dégager de la perspective habermassienne des publics « rationnels-critiques », échouant à cerner leurs propositions en termes de nouveaux styles de langage et registres de communication, voire d'esthétique de la contestation. C'est cet aspect que développe tout particulièrement Konstantinos Eleftheriadis dans ce dossier, avec une contribution portant sur les pratiques militantes liées à l'organisation de festivals queer européens. Des théoriciennes féministes ont plaidé pour la prise en considération de registres d'intervention alternatifs dans l'espace public, tels que la narration (Mansbridge, 1999) ou le récit personnel (Young, 1996), voire le sit-in, le boycott, le chant (Butler, Spivak, 2007) ou toute forme de rassemblement public troublant les formes de reconnaissance du politique et leurs exclusions constitutives (Young, 201 I ; Butler, 20 I5). Cette revalorisation de l'action directe plaide que celle-ci serait la seule à même de dévoiler les consensus factices (préjugés ou croyances au caractère inaltérable de telle ou telle réalité sociale) engendrés par les discours hégémoniques sur lesquels reposent les procédures de délibération (Young, 201 I). 
Sur ce point, tout particulièrement, l'approche communicationnelle offre des outils précieux pour mettre au jour la normativité des modalités d'intervention dans le débat public, que dénoncent les théories féministes et queer de l'espace public, dans la mesure où cette approche fournit des outils conceptuels pour étudier la manière dont les rapports de pouvoir travaillent les dispositifs de médiation" (Julliard, 2009). La relecture en SIC de la conceptualisation du dispositif par Michel Foucault et Gilles Deleuze, notamment pour appréhender les dispositifs d'écriture numérique (par exemple Bonaccorsi, Julliard, 2010 ; Monnoyer-Smith, 20I 3 ; Badouard, Mabi, Monnoyer-Smith,20 I 6), constitue ainsi un cadre d'appréhension favorable des formes de participation aux débats ${ }^{12}$ autant que des formations discursives autorisées, de la reproduction des rapports de domination, ou encore des lieux de mise en visibilité de certaines subjectivations (Julliard, 2016). La contribution de Florian Vörös au dossier chemine également à partir de la relecture par Gilles Deleuze de la conceptualisation foucaldienne du dispositif pour étudier l'imbrication des rapports de pouvoir dans des dispositifs de débat télévisé mettant en scène un affrontement entre différentes définitions du racisme (et de l'antiracisme). Le croisement d'une telle approche communicationnelle avec les perspectives développées au sein des études de genre est non seulement heuristique, mais permet surtout de réintroduire une attention à la conflictualité sociale là où le recours courant au concept de dispositif en SIC a pu aussi parfois conduire à sa dépolitisation (Gavillet, 20 I0).

\section{Conclusion}

Au-delà de leurs différences d'appréciation du processus délibératif et de ses limitations, la théorie queer et les théories féministes y compris d'inspiration francfortienne convergent toutefois sur un point, celui de la nécessité de rendre compte de la co-constitution et des interactions entre les différents axes de différenciation sociale. Ceci trouve une résonnance particulière dans le projet politique queer de refus de l'assimilation des minorités sexuelles, celle-ci étant perçue comme impliquant une légitimation et un renforcement des inégalités liées aux rapports de classe et/ou à la racialisation du social (Warner, 1993 ; Duggan, 2003 ; Bassichis, Spade, 20 I4). Au sein des théories féministes, le principe de modélisation des relations qu'entretiennent le genre, la sexualité, la classe et la race a donné lieu à de multiples outils analytiques, bien que durant les deux dernières décennies les débats se soient essentiellement focalisés sur le concept

\footnotetext{
" Au sujet de l'approche communicationnelle utilisée pour appréhender les rapports de pouvoir qui travaillent les dispositifs de médiation voir, par exemple, les travaux menés au Centre de recherche sur les médiations (Crem, université de Lorraine) sur les notions de « (dispositifs de) médiation » (notamment Aghababaie et al., 2010 ; Appel, Boulanger, Massou, 2010).

${ }^{12}$ Ainsi que le souligne Laurence Monnoyer-Smith (2013:25), c'est « précisément parce que le dispositif cadre la participation que certains refusent d'y entrer, considérant qu'lls participent à la construction d'une légitimité procédurale à laquelle ils refusent d'adhérer (Mouffe, 1999) ».
} 
d'intersectionnalité forgé par Kimberlé Crenshaw ( 199 |), sa dimension heuristique, ses fondements épistémologiques et ses limites dans l'application empirique ${ }^{13}$ (Bilge, 2009 ; Hill Collins, Bilge, 2016 ; Fassa, Lépinard, Roca i Escoda, 2016). La plupart des contributions de ce dossier s'attachent ainsi à étudier l'articulation de ces axes de différenciation sociale dans les modalités de médiatisation d'une controverse. C'est notamment le cas de celle de Marion Dalibert qui donne à voir la façon dont la réception médiatique du roman d'Édouard Louis Pour en finir avec Eddy Bellegueule participe, à partir d'un discours sur l'expression de genre et la sexualité du protagoniste, de la constitution des « classes populaires blanches du nord de la France » en figure de l'altérité, en particulier dans la presse nationale. Comme le montrent ces contributions, les relations entre les principaux axes de différenciation sociale se manifestent dans le discours médiatique sur un mode très complexe. Le marquage de la différence prend une forme distincte s'agissant de la construction du genre, de la racialisation, du partage des sexualités ou de la production d'un ordre symbolique de classe. II n'est cependant pas rare que le genre ou le rapport à la sexualité opèrent comme des vecteurs de racialisation ${ }^{14}$, ou qu'une classe sociale se voit attribuer des caractéristiques stéréotypées en termes d'identité de genre. Autrement dit, les principaux axes de différenciation qui sous-tendent les rapports sociaux se trouvent imbriqués sur un mode complexe dans les représentations médiatiques et dans le débat public. Dès lors, si l'on souhaite faire émerger leur articulation, il s'agit de prêter attention aux non-dits des discours, aux principes de division silencieux qui les sous-tendent, à la charge affective qui les traverse, voire à la manière dont un processus de segmentation du social s'exprime dans les termes d'un autre.

\section{Références}

Achin C. et al., dirs, 2007, Sexes, genre et politique, Paris, Éd. Economica.

Aghababaie M. et al., dirs, 20 I 0, Usages et enjeux des dispositifs de médiation, Nancy, Presses universitaires de Nancy.

Allen A., 1988, Uneasy Access. Privacy for Women in a Free Society, Lanham, Rowman \& Littlefield.

Amato É. A., Pailler F., SchaferV., dirs, 2014, « Sexualités 》, Hermès. La Revue, 69, 2. Accès : https://www.cairn.info/revue-hermes-la-revue-20|4-2.htm.

\footnotetext{
${ }^{13}$ La question des usages du concept d'intersectionnalité dans les enquêtes de terrain a été particulièrement traitée lors de la journée d'étude organisée par le Domaine d'intérêt majeur de la Région Île-de-France «"Genre, inégalités, discriminations" : L'intersectionnalité en pratiques : disciplines, méthodes et enquêtes », qui s'est déroulée à l'Institut national d'études démographiques (Ined) le 14 novembre 2014 (http://wnw.gid-idf.org/fr/activites/view/29//-intersectionnalite-en-pratiquesdisciplines-methodes-et-enquetes).

${ }^{14}$ Sur le genre comme opérateur de racialisation, voir par exemple le travail de Marion Dalibert (2014) sur la médiatisation des Roms et des habitants de banlieue.
} 
AppelV., Boulanger H., Massou L., dirs, 2010 , Les Dispositifs d'information et de communication. Concepts, usages et objets, Bruxelles, De Boeck.

Badouard R., Mabi C., Monnoyer-Smith L., 2016, « Le débat et ses arènes. À propos de la matérialité des espaces de discussion », Questions de communication, 30, pp. 7-23. Accès : https://www.cairn.info/revue-questions-de-communication-20 I 6-2-page-7.htm.

Bassichis M., Spade, D., 20 I4, « Queer politics and anti-blackness », pp. 191-2 10, in : Haritaworn J., Kuntsman A., Posocco S., dirs, Queer Necropolitics, New York, Routledge.

Benoît A., 20 I 4, « L'"espace public" à l'épreuve de la critique féministe », Philonsorbonne, 8, pp. | 2 | - | 3 | . Accès : http://journals.openedition.org/philonsorbonne/576.

Berlant L., Warner M., 1998, « Sex in Public », Critical Inquiry, 24, 2, pp. 547-566.

Berthelot-Guiet K., Kunert S., dirs, 20 I 3, «Les langages du genre:sémiotique et communication », Communication \& Langages, 177. Accès : https://www.cairn.info/revue-communication-etlangages |-20|3-3.htm.

Bilge S., 2009, «Théorisations féministes de l'intersectionnalité », Diogène, 225, I, pp. 70-88. Accès : https://www.cairn.info/revue-diogene-2009-I-page-70.htm.

Bonaccorsi J., Julliard V., 20I0, « Dispositifs de communication numériques et médiations du politique. Le cas du site Web d'Ideal-Eu », pp. 65-78, in : Aghababaie M. et al., dirs, Usages et enjeux des dispositifs de médiation, Nancy, Presses universitaires de Nancy.

Bonnafous S., Jouët J., Rieffel R., dirs, 2003, « Une communication sexuée? », Réseaux, 120, 4. Accès : https://www.cairn.info/revue-reseauxI-2003-4.htm.

Bouchard J., Froissart P., dirs, 2004, « Sexe \& Communication », MEI, 20.

Butler J., 1990, Trouble dans le genre. Le féminisme et la subversion de l'identité, trad. de l'anglais par C. Kraus, Paris, Éd. La Découverte, 2006.

Butler J., 1993, Ces corps qui comptent. De la matérialité et des limites discursives du « sexe », trad. de l'anglais par C. Nordmann, Paris, Amsterdam, 2009.

Butler J., 1997, « Simplement culturel ? », trad. de l'anglais par B. Marrec, pp. I 68- I 83, in : BidetMordrel A., dir., Les Rapports sociaux de sexe, Paris, Presses universitaires de France, 2010.

Butler J., 20 15, Rassemblement. Pluralité, performativité et politique, trad. de l'anglais par C. Jaquet, Paris, Fayard, 2016.

Butler J., Spivak G. C., 2007, L'État global, trad. de l'anglais par F. Bouillot, Paris, Payot, 2009.

Cervulle M., 20 I4, « La sexualisation normative de l'espace public », Hermès. La Revue, 69, pp. |46- I 5 I. Accès : https://www.cairn.info/revue-hermes-la-revue-20 | 4-2-page- | 46.htm.

Cervulle M., 2017, «Exposer le racisme. Exhibit B et le public oppositionnel », Études de communication, 48, pp. 37-54.

Cervulle M., JulliardV., 2013, «"Différence des sexes" et controverses médiatiques. Du débat sur la parité au "mariage pour tous" (1998-20 I3) », Le Temps des médias, 2 I, 2, pp. I 6 I - 175.

Cervulle M., Pailler F., 2014, « \#mariagepourtous: Twitter et la politique affective des hashtags », Revue française des sciences de l'information et de la communication, 4. Accès : http://journals.openedition.org/rfsic/717.

Chauvel S., Le Renard A., 20 13, « Comment le travail journalistique amplifie la hiérarchie de genre. Une rédaction pendant la campagne présidentielle de 2012 », Genre, sexualité \& société, hors-série, 2, Accès : http://journals.openedition.org/gss/2598. 
Clarke E. O., 2000, Virtuous Vice. Homoeroticism and the Public Sphere, Durham, Duke University Press.

Constantin de Chanay H., ChevalierY., Gardelle L., dirs, 20 I7, «Écrire le genre », Mots. Les langages du politique, 113.

Coulomb-Gully M., Bonnafous S., dirs, 2007, « Femmes et médias : le 8 mars à la "une" », Sciences de la société, 70.

Coulomb-Gully M., dir., 201 I, « Médias : la fabrique du genre », Sciences de la société, 83. Accès : http://journals.openedition.org/sds/2009.

Coulomb-Gully M., Méadel C., 201 I, « Plombières et jardinières. Résultats d'enquêtes et considérations méthodologiques sur la représentation du genre dans les médias », Sciences de la société, 83, pp. 15-35. Accès : http://journals.openedition.org./sds/21 I 5.

Crenshaw K., 1991, « Cartographie des marges : intersectionnalité, politique de l'identité et violences contre les femmes de couleur », trad. de l'anglais par O. Bonis, Cahiers du genre, 39, 2005, pp. 5 I-82. Accès : https://www.cairn.info/revue-cahiers-du-genre-20052-page-5I.htm.

Dalibert M., 2013, «Authentification et légitimation d'un problème de société par les journalistes : les violences de genre en banlieue dans la médiatisation de Ni putes ni soumises 》, Études de communication,40, pp. 167- | 80. Accès : http://journals.openedition. org/edc/52| 4 .

Dalibert M., 2014, « Le marquage socio-discursif de la race par le genre. Les "roms", les Tunisiens, les Ukrainiens et les habitants des banlieues françaises dans les médias », Revue française des sciences de l'information et de la communication, 4. Accès : http:// journals.openedition.org/rfsic/743.

Dalibert M., Quemener N., 2016, « Femen. La reconnaissance médiatique d'un féminisme aux seins nus », Mots. Les langages du politique, I I I, pp. 83- 102.

Damian-Gaillard B., Montañola S., Olivesi A., dirs, 2014, L'Assignation de genre dans les médias. Attentes, perturbations, reconfigurations, Rennes, Presses universitaires de Rennes.

Damian-Gaillard B., Saïtta E., 201 I, « Le processus de féminisation du journalisme politique et les réorganisations professionnelles dans les quotidiens nationaux français », Communication, 28, 2. Accès : http://journals.openedition.org/communication/l 725.

De LauretisT., 1987, « La technologie du genre », pp. 37-94, in : De LauretisT., Théorie queer et cultures populaires. De Foucault à Cronenberg, trad. de l'anglais par M.-H. Bourcier, Paris, Éd. La Dispute, 2007.

Delphy C., 200 I, « Penser le genre : problèmes et résistances », pp. 243-260, in : Delphy C., L'Ennemi principal, vol. 2 : Penser le genre, Paris, Éd. Syllepse.

Derrida J., 1989, Limited Inc., trad. de l'anglais par E.Weber, Paris, Galilée, 1990.

Desmarchelier D., Rennes J., dirs, 2005, « Usages politiques du genre », Mots. Les langages du politique, 78. Accès : http://journals.openedition.org/mots/67.

Dorlin E., 2005, « Sexe, genre et intersexualité : la crise comme régime théorique », Raisons politiques, 18, 2, pp. I 17-137. Accès : https://www.cairn.info/revue-raisons-politiques-20052-page- $117 . \mathrm{htm}$.

Duggan L., 2003, The Twilight of Equality? Neoliberalism, Cultural Politics, and the Attack on Democracy, Boston, Beacon Press. 
Dulong D., Matonti F., 2005, « L'indépassable féminité. La mise en récit des femmes en campagne », pp. 28 I-303, in : Lagroye J., Lehingue P., Sawicki F., dirs, Mobilisations électorales. Le cas des élections municipales de 2001 , Paris, Presses universitaires de France.

Elshtain J. B., 1981, Public Man, Private Woman. Women in Social and Political Thought, Princeton, Princeton University Press.

Engels F., I884, L'Origine de la famille, de la propriété privée et de l'État, trad. de l'allemand par J. Stern, Paris, Éd. Sociales, 1954.

Espineira K., 2014, « La médiatisation des politiques transgenres : du statut de contrepublic à l'inégalité de la représentation », Revue française des sciences de l'information et de la communication, 4. Accès : http://journals.openedition.org/rfsic/695.

Espineira K., 20I5, Médiacultures. La transidentité en télévision, Paris, Éd. L'Harmattan.

Fassa F., Lépinard E., Roca i Escoda M., dirs, 2016, L'Intersectionnalité. Enjeux théoriques et politiques, Paris, Éd. La Dispute.

Fleury B.,Walter J., dirs, 2005, « Espaces politiques au féminin », Questions de communication, 7. Accès : http://journals.openedition.org/questionsdecommunication/4040.

Fleury B., Walter J., dirs, 2009a, « Penser le genre en sciences de l'information et de la communication et au-delà », Questions de Communication, I 5, pp. I I I-260. Accès : http:// journals.openedition.org/questionsdecommunication/424.

Fleury B., Walter J., dirs, 2009b, « Penser le genre en sciences de l'information et de la communication et au-delà (2) », Questions de Communication, 16, pp. I31-210. Accès : http://journals.openedition.org/questionsdecommunication/59.

Fleury B., Walter J., 2017, « Les valeurs : quelles théories, quelles méthodes? », Questions de communication, 32, pp. I53-166.

Floyd K., 2009, La Réification du désir. Vers un marxisme queer, trad. de l'anglais par M. Dennehy et al., Paris, Amsterdam, 2013.

Foucault M., 1976, Histoire de la sexualité. Tome I. La volonté de savoir, Paris, Gallimard.

Fraser N., 1985, Le Féminisme en mouvements. Des années 1960 à l'ère néolibérale, trad. de l'anglais par E. Ferrarese, Paris, Éd. La Découverte, 2012.

Fraser N., 1992, «Repenser la sphère publique : une contribution à la critique de la démocratie telle qu'elle existe réellement », trad. de l'anglais par M.Valenta, Hermès. La Revue, 31, 3, 2001, pp. 125-156.

Fraser N., 1997, « Heterosexism, Misrecognition and Capitalism: A Response to Judith Butler », Social Text, 52-53, pp. 279-289.

Fraser N., 2005, Qu'est-ce que la justice sociale? Reconnaissance et redistribution, trad. de l'anglais par E. Ferrarese, Paris, Éd. La Découverte, 201 I.

Fraser N., 2010, «Pour une politique féministe à l'âge de la reconnaissance : approche bi-dimensionnelle et justice entre les sexes », trad. de l'anglais par B. Marrec, pp. |23-|4I, in : Bidet-Mordrel A., dir., Les Rapports sociaux de sexe, Paris, Presses universitaires de France.

Gavillet I., 2010, « Michel Foucault et le dispositif: questions sur l'usage galvaudé d'un concept », pp. 17-38, in :AppelV., Boulanger H., Massou L., dirs, Les Dispositifs de l'information et de la communication. Concepts, usages et objets, Bruxelles, De Boeck.

Göle N., 1997, «The Gendered Nature of the Public Sphere », Public Culture, 10, I, pp. 6I -81. 
Habermas J., 1962, L’Espace public. Archéologie de la publicité comme dimension constitutive de la société bourgeoise, trad. de l'allemand par M. B. de Launay, Paris, Payot, 1993.

Heinich N., 2017, « Dix propositions sur les valeurs », Questions de communication, 31, pp. 291-313.

Hernández Orellana M., Kunert S., 20 I4, Quand l'État parle des violences faites aux femmes, Paris, Lussaud.

Hill Collins P., Bilge S., 20I6, Intersectionality, Cambridge, Polity press.

Honneth A., 1992, La Lutte pour la reconnaissance, trad. de l'allemand par P. Rusch, Paris, Éd. Le Cerf, 2000.

Julliard V., 2009, « Pour une intégration du genre par les sciences de l'information et de la communication », Questions de communication, 16, pp. 191-210. Accès: http://journals. openedition.org/questionsdecommunication/356.

Julliard V., 20 I2, De la presse à Internet. La parité en questions, Paris, Lavoisier.

JulliardV., 20 I 6, « \#Theoriedugenre : comment débat-on du genre surTwitter? », Questions de communication, 30, pp. 135-157.

JulliardV., 2017, «"Théorie du genre", "\#theoriedugenre": stratégies discursives pour soustraire la "différence des sexes" des objets de débat », Études de communication, 48, pp. | | | - 136.

Julliard V., Quemener N., dirs, 20 I4, « Émergence : le genre dans la communication et les médias », Revue française des sciences de l'information et de la communication, 4. Accès: http://journals.openedition.org/rfsic/69l.

Kaufmann L., Gonzalez P., 2017, « Mettre en valeur(s) le monde social », Questions de communication, 32, pp. 167-194.

Kraus C., 2000, « La bicatégorisation par "sexe" à l'épreuve de la science : le cas des recherches en biologie sur la détermination du sexe chez les humains », pp. |87-2।3, in : Gardey D., Löwy l., dirs, L'Invention du naturel. Les sciences et la fabrication du féminin et du masculin, Paris, Éd. des Archives contemporaines.

Lécossais S., 2014, « Les mères ne sont pas des parents comme les autres. Genre et parentalité dans les séries télévisées françaises », Revue française des sciences de l'information et de la communication, 4. Accès : http://journals.openedition.org/rfsic/706.

Lemieux C., 2007, « À quoi sert l'analyse des controverses? », Mil neuf cent. Revue d'histoire intellectuelle, 25, pp. 191-212. Accès : https:/www.cairn.info/revue-mil-neuf-cent-2007I-page- $191 . \mathrm{htm}$.

Lunt P., Livingstone S., 1994, « Se faire entendre dans l'espace public. Les femmes, la télévision et le citoyen-téléspectateur », trad. de l'anglais par É. Maigret, Réseaux, 63, pp. 59-74. Accès : https://www.persee.fr/doc/reso_075 I-797I_1994_num_12_63_2435.

Mansbridge J., 1999, « Everyday talk in the Deliberative System », pp. 21 I-239, in : Macedo S., dir., Deliberative Politics. Essays on democracy and disagreement, Oxford, Oxford University Press.

Marignier N., 20 I 6, Les Matérialités discursives du sexe. La construction et la déstabilisation des évidences du genre dans les discours sur les sexes atypiques, thèse en sciences du langage, Université Paris 13.

Martuccelli D., 2017, « La grammaire axiologique et la sociologie des valeurs », Questions de communication, 32, pp. 195-218. 
Marx K., Engels F., 1846, L'Idéologie allemande, trad. de l'allemand par H. Auger et al., Paris, Éd. Sociales, 1968.

Monnoyer-Smith L., 20 I3, « Le web comme dispositif: comment appréhender le complexe? », pp. I I-3I, in : Barats C., dir, Manuel d'analyse du web en sciences humaines et sociales, Paris, A. Colin.

Mouffe C., 1999, « Deliberative democracy or agonistic pluralism ? », Social Research, 66, 3, pp. 745-758.

Olivesi A., 20 I2, Implicitement sexiste? Genre, politique et discours journalistique, Toulouse, Presses universitaires du Mirail.

Pailler F., 201 I, « Chatroulette, et le sexe devint l'erreur du web 2.0 », POLI. Politiques de l'image, 4, pp. 8I-96.

Pateman C., 1988, Le Contrat sexuel, trad. de l'anglais par C. Nordmann, Paris, Éd. La Découverte, 2010.

Paveau M.-A.,PereaF.,dirs,20 I 4, «Lapornographieetses discours», Questions de communication, 26. Accès : http://journals.openedition.org/questionsdecommunication/9194.

Quemener N., 2014, Le Pouvoir de l'humour. Politiques des représentations dans les médias en France, Paris, A. Colin/Ina Éd.

Quéré L., 20 17, « Comment "pragmatiser" le champ de la valeur », Questions de communication, 32, pp. 195-218.

Rendall J., I 999, «Women and the Public Sphere », Gender and History, I I, 3, pp. 475-488.

TavernierA., 2004, « De la parité de genre à l'égalité des sexes. La construction d'un référentiel médiatique », MEl, 20, pp. | 49- 158. Accès : http://www.mei-info.com/wp-content/uploads/ revue20/ilovepdf.com_split_15.pdf.

Taylor C., 1994, Multiculturalism. Examining the Politics of Recognition, Princeton, Princeton University Press.

Vörös F., 2015, Les Usages sociaux de la pornographie en ligne et les constructions de la masculinité : une sociologie matérialiste de la réception des médias, thèse en sociologie, École des hautes études en sciences sociales.

Warner M., 2005, Publics and Counterpublics, New York, Zone Books.

Warner M., dir., 1993, Fear of a Queer Planet. Queer Politics and Social Theory, Minneapolis, University of Minnesota Press.

Young I. M., 1990, Justice and the Politics of Difference, Princeton, Princeton University Press.

Young I. M., 1996, « Communication and the Other: Beyond Deliberative Democracy », pp. I20-135, in : Benhabib S., dir., Democracy and Difference. Contesting the Boundaries of the Political, Princeton, Princeton University Press.

Young I. M., 200I, « La démocratie délibérative à l'épreuve du militantisme », trad. de l'anglais par M. Saint-Upéry, Raisons politiques, 42, 201 I, pp. I31-158. Accès : https:// www.cairn.info/revue-raisons-politiques-20 I I-2-page- 131 .htm. 